\title{
Expressive aphasia caused by Streptococcus intermedius brain abscess in an immunocompetent patient
}

\author{
Misbahuddin Khaja' \\ Darryl Adler ${ }^{2}$ \\ George Lominadze ${ }^{2}$ \\ 'Division of Pulmonary and Critical \\ Care Medicine, Bronx Lebanon \\ Hospital Center, Affiliated with Icahn \\ School of Medicine at Mount Sinai, \\ ${ }^{2}$ Division of Critical Care Medicine, \\ New York Presbyterian-Lawrence \\ Hospital Center, Affiliated with \\ Columbia University College of \\ Physician and Surgeons, Bronx, \\ New York, NY, USA
}

This article was published in the following Dove Press journal: International Medical Case Reports Journal

23 January 2017

Number of times this article has been viewed
Background: Brain abscess is an uncommon but life-threatening infection. It involves a focal, intracerebral infection that begins in a localized area of cerebritis and develops into a collection of pus, surrounded by a well-vascularized capsule. Brain abscess still poses a significant problem in developing countries but rarely in developed countries. Predisposing factors vary in different parts of the world. With the introduction of antibiotics and imaging studies, the mortality rate has decreased between $5 \%$ and $15 \%$. If left untreated it may lead to serious neurologic sequelae. The temporal lobe abscess can be caused by conditions like sinusitis, otitis media, dental infections, and mastoiditis if left untreated or partially treated. Additionally, in neurosurgical procedures like craniotomy, the external ventricular drain can get infected, leading to abscess formation.

Case presentation: We present the case study of an elderly female patient who presented with expressive aphasia caused by brain abscess, secondary to Streptococcus intermedius infection. The 72-year-old female with a medical history of hypertension came to hospital for evaluation with word-finding difficulty, an expressive aphasia that began a few days prior to presentation. Computed tomography of the head showed a left temporal lobe mass-like lesion, with surrounding vasogenic edema. The patient was empirically started on courses of antibiotics. The next day, she was subjected to magnetic resonance imaging of the brain, which showed a left temporal lobe septated rim-enhancing mass lesion, with bright restricted diffusion and diffuse surrounding vasogenic edema consistent with abscess. The patient was also seen by the neurosurgery department and underwent stereotactic, left temporal craniotomy, with drainage, and resection of abscess. Tissue culture grew S. intermedius sensitive to ampicillin sulbactam. Subsequently her expressive aphasia improved.

Conclusion: Brain abscess has a high mortality, however a significant proportion of patients with appropriately treated abscess recover completely and can survive without significant neurologic damage. Advanced imaging modalities may yield more accurate methods of differentiation of mass lesions in the brain. Biopsy of brain lesion with early initiation of appropriate antibiotics will change the outcome.

Keywords: expressive aphasia, Streptococcus intermedius, brain abscess

\section{Introduction}

There are $\sim 1,500-2,000$ cases of brain abscess diagnosed annually in the USA, ${ }^{1}$ resulting in an estimated 1 in 10,000 individuals being hospitalized for this condition every year. Critically, the all-cause mortality rate in patients hospitalized with brain abscess varies from $5 \%$ to $32 \% .^{2}$ Brain abscess is rare in developed countries but still has significant problems in developing countries. ${ }^{3}$

Temporal lobe abscess is a rare and dangerous complication secondary to mastoiditis, an infection in the mastoid air cells. This can be caused by both Grampositive and Gram-negative organisms, including those from the Nocardia, Listeria,
Correspondence: Misbahuddin Khaja Medicine, Bronx Lebanon Hospital Center, Affiliated with Icahn School of

Tel + I 3479677074

Email drkhaja@yahoo.com

Dovepress in


Corynebacterirum, Actinomyces, Rhodococcus, Mycobacterium, Peptostreptococcus, Fusobacterium, and Bacteroides genera, as well as fungal and parasitic organisms. ${ }^{4}$

A major risk factor for brain abscess is the presence of a contiguous focus of infection, due to such conditions as sinusitis, otitis media, mastoiditis, dental infection, infective endocarditis, lung abscess, neurosurgical procedure, and head trauma. ${ }^{5}$ Other risk factors include an immunosuppressed state, such as observed in acquired immunodeficiency syndrome or the drug-induced immunosuppression required in solid organ and bone marrow transplant recipients. ${ }^{6}$

Streptococcus anginosus is the taxonomically accepted name for a group of streptococcal species that includes $S$. anginosus, Streptococcus intermedius, and Streptococcus constellatus. Before these three strains were collectively known as $S$. anginosus, they were formerly, and collectively, known as the single species, Streptococcus milleri. ${ }^{7}$ Members of the $S$. anginosus group possess proteolytic enzymes that cause necrosis of tissue and promote abscess formation. ${ }^{8}$

\section{Case report}

A 72-year-old female with a medical history of hypertension came to hospital for evaluation with word-finding difficulty, an expressive aphasia that began a few days prior. She denied headache, weakness, or blurred vision.

In the Emergency Department, she was found to have a fever of $38.3^{\circ} \mathrm{C}$, a heart rate of $96 \mathrm{bpm}$, and a blood pressure of 126/74 $\mathrm{mmHg}$. On physical examination, she was alert, oriented to both self and place, and date and year. Her lungs were clear, and the dental examination was normal, with no lymphadenopathy. Naming, however, was impaired; she replied "football" when asked to name a stethoscope and answered "sunglasses" when shown a pair of glasses.

The patient was able to spell the word "world" in the forward direction and made one mistake when spelling it backwards. Registration was $3 / 3$, recall was $2 / 3$, and both remote and recent memory were intact. Her speech was also fluent and coherent, and the cranial nerves were intact. She displayed a shoulder shrug of $5 / 5$, and the strength in both the upper and lower extremities was $5 / 5$. Tone and bulk were normal, and reflexes were in the 1 range. Toes were downgoing, and coordination was intact for both finger-to-nose and toe-to-finger. Sensation was intact to light touch, pinprick, vibration, and proprioception. Her gait was normal, steady, and narrow-based.

Computed tomography (CT) of the head (Figure 1) showed a left temporal lobe mass-like lesion, with surrounding vasogenic edema. Our differential diagnoses were wide, ranging from metastatic disease, brain abscess, cerebral

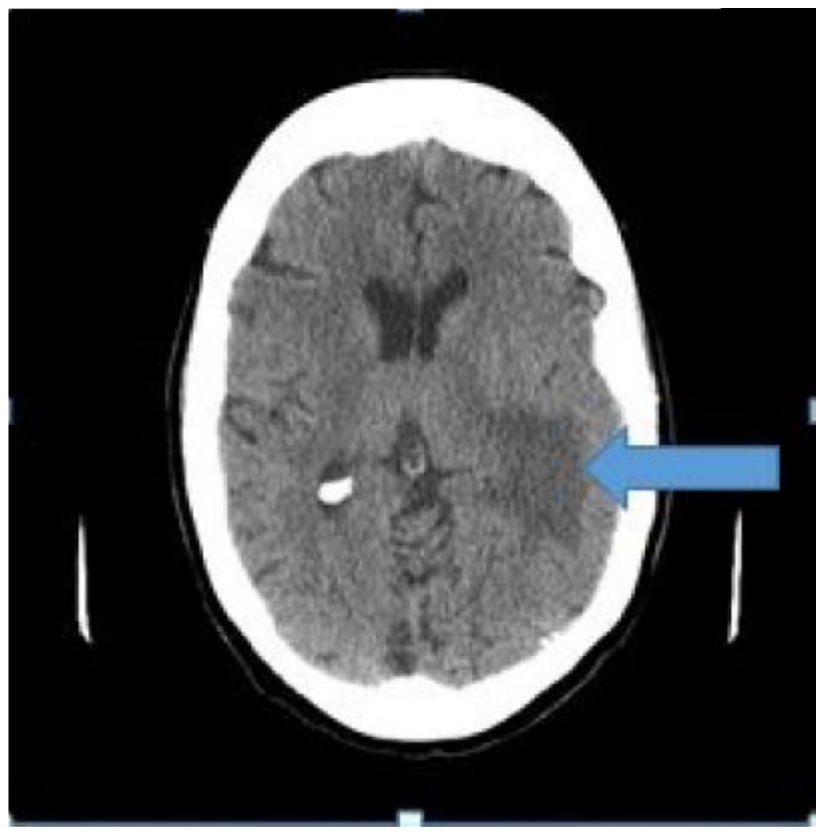

Figure I Computed tomography of the head shows a left temporal lobe mass-like lesion, with surrounding vasogenic edema (arrow).

infarction, lymphoma, high grade glioma, toxoplosmosis, demyelinating disease, or resolving cerebral contusion/ hematoma.

The patient was empirically started on courses of vancomycin, ampicillin, and ceftriaxone antibiotics. The next day, she was subjected to magnetic resonance imaging (MRI) of the brain with gadolinium contrast (Figure 2), which showed

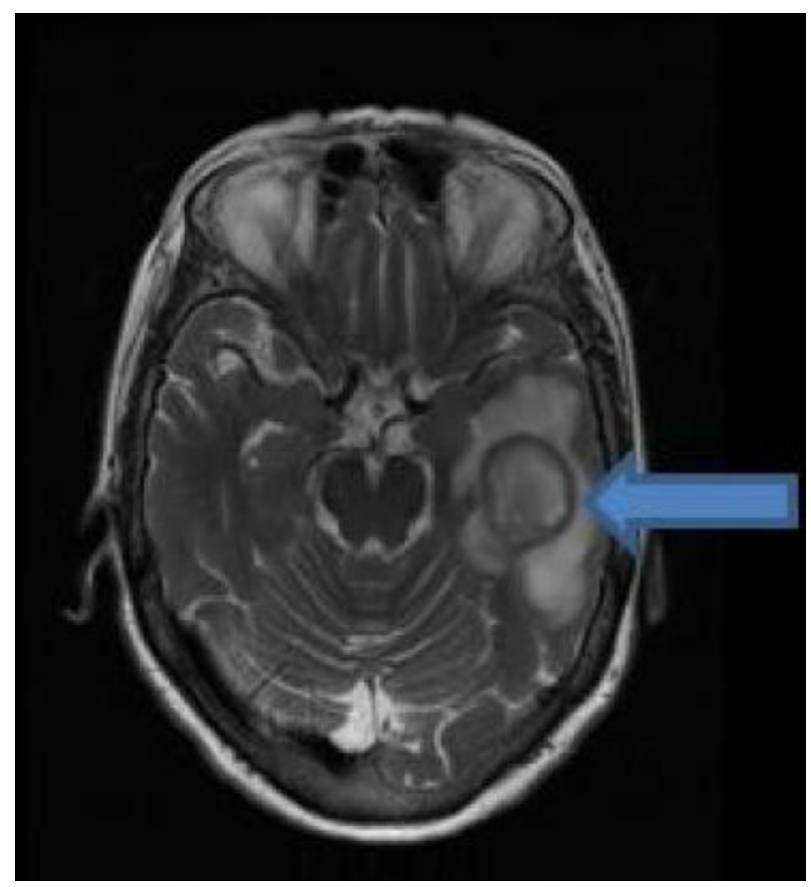

Figure 2 Magnetic resonance imaging of the brain shows a septated rim-enhancing mass lesion in the left temporal lobe, with bright, restricted diffusion, and diffuse surrounding vasogenic edema (arrow). 
a left temporal lobe septated rim-enhancing mass lesion, with bright restricted diffusion and diffuse surrounding vasogenic edema. Primary consideration was an abscess, especially with a dark rim of T2 signal, although other possibilities were also considered, including cystic glioblastoma, metastatic carcinoma, and lymphoma.

Laboratory findings revealed a white cell count of $11.3 \mathrm{~K} / \mathrm{UL}$; her human immunodeficiency virus test was negative, and echocardiogram ejection fraction was $65 \%$, with no vegetation. Her chest radiograph showed no evidence of infection. Transesophageal echocardiogram was also done and showed no evidence of vegetation. The patient was seen by dental services and all teeth were found to be intact, with no evidence of decay or infection.

The patient was also examined by the neurosurgery department and underwent stereotactic, left temporal craniotomy, with drainage and resection of abscess. Tissue culture grew $S$. intermedius. Brain biopsy showed abundant acute inflammatory cells (neutrophils) and necrotic materials, consistent with abscess (Figure 3). As per the known sensitivity of $S$. intermedius, antibiotics were deescalated to intravenous ampicillin sulbactum.

The patient was discharged to home on the ninth postoperative day, while continuing a course of intravenous antibiotics with the help of visiting nurse services. Our patient received 4 weeks of intravenous antibiotics as outpatient. Her expressive aphasia began to improve, and follow-up MRI of the brain (Figure 4) performed after 2 months showed left-sided craniotomy changes with interval collapse of the postoperative cavity and decreased surrounding edema and enhancement. The patient completely recovered from the expressive aphasia; however, the source of the pathogen was not detected, despite extensive examination.

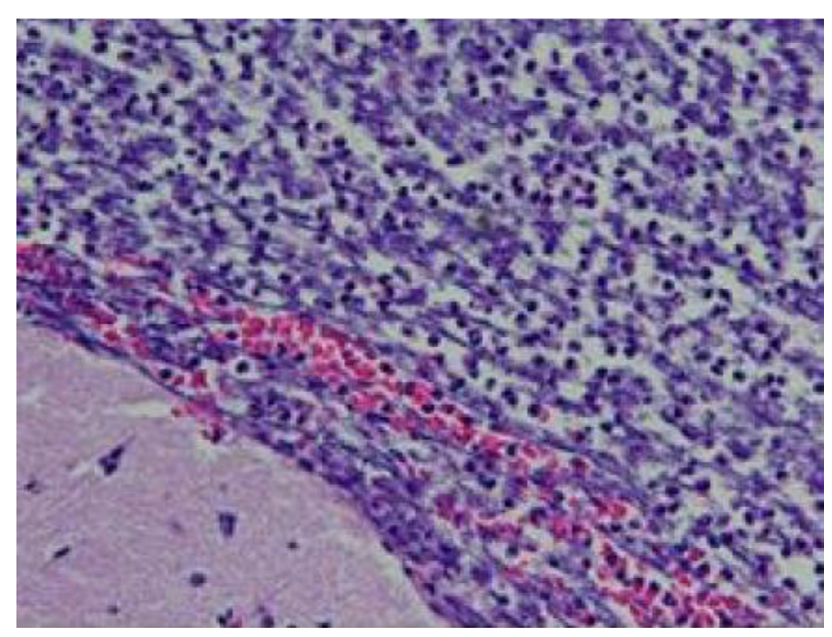

Figure 3 Abundant neutrophils and necrotic debris.

Note: Minute fragment of brain tissue is visible in the left lower corner (hematoxylin \& eosin, original magnification $20 \times$ ).

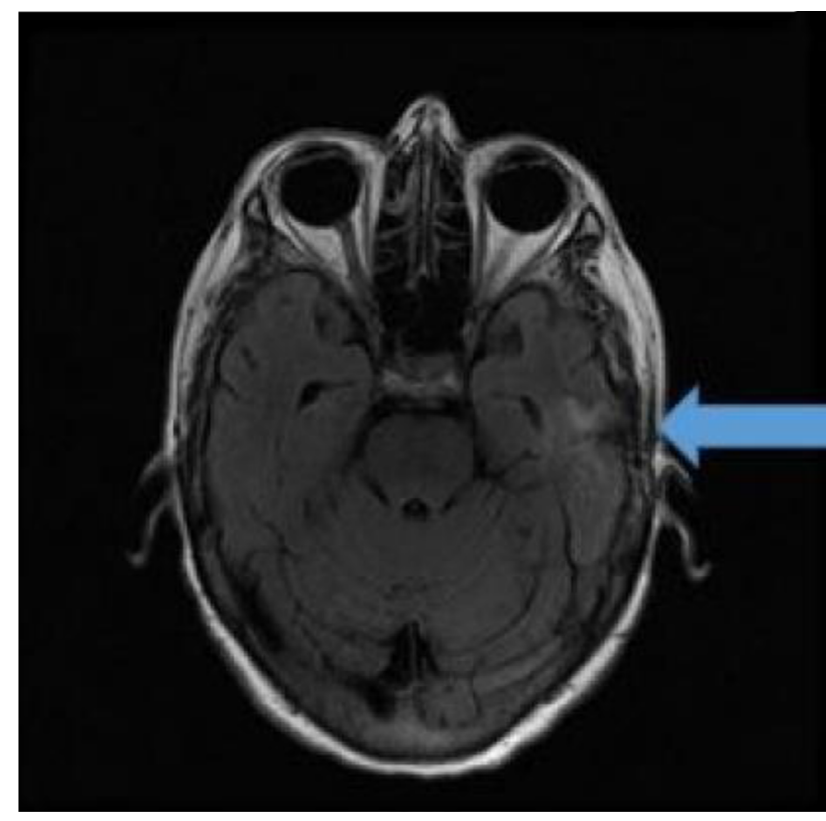

Figure 4 Two months follow-up: magnetic resonance imaging of the brain shows left-sided craniotomy changes with interval collapse of postoperative cavity and decreased surrounding edema and enhancement (arrow).

Our case is unique as our patient was immunocompetent, she did not have any ear infection, dental infection, no recent head trauma, no surgeries, and echocardiogram was negative for any endocarditis. Nor she received chemotherapy or corticosteroids.

Patient has given written informed consent for publication of this case, including images. Consent is available with corresponding author (Misbahuddin Khaja).

\section{Discussion}

The first case study describing an association between S. intermedius and deep-seated purulent abscess was reported in 1975. ${ }^{9}$ These nonhemolytic viridans streptococci were described by Guthof in 1956 and were originally named $S$. milleri. ${ }^{10}$ The official name for this heterogeneous group of organisms has now been changed to $S$. anginosus, and it includes three species: S. anginosus, S. intermedius, and $S$. constellatus. Most common causes of brain abscess are viridans streptococci and Staphylococcus aureus. S. intermedius is found in normal flora of oral cavity, gastrointestinal tract, and upper airways; in contrast to other pathogenic streptococci, they are also associated with abscess formation. ${ }^{4}$

Several mechanisms have been proposed to explain the spread from a contiguous focus of infection to the brain: these include, extension from sinuses complicated by osteomyelitis, spread of local infection to the brain via emissary veins of the skull, retrograde spread from the vasculature of the sinus mucosa through the valveless network of veins into the cerebral venous system, spread via lymphatics, and 
postneurosurgery infection resulting from deep inoculation into the brain. Pecharki et al further showed that hyaluronidase, an enzyme that breaks down host tissues and allows the bacteria to utilize the products for growth, plays a role in the dispersal of $S$. intermedius biofilms. ${ }^{11}$

Patients with brain abscess often present with headache, vomiting, neck stiffness, change in mental status, seizures, hemiparesis, loss of vision, and dysphagia. ${ }^{12}$ Stewart et al reported a case of a 74-year-old immunocompetent man admitted for severe retro-orbital headache, who was diagnosed with isolated sphenoiditis and temporal lobe abscess cultures showing $S$. anginosus. ${ }^{13}$ Chen et al described 49-year-old female with headache, who was found to have two temporal lobe abscesses, the cultures from which grew Pseudomonas aeruginosa and Stenotrophomonas maltophilia. ${ }^{14}$ Guzman et al reported the case of a 24-year-old female who presented with right-sided otalgia, otorrhea, and hearing loss; CT scans from this patient showed temporal lobe abscess. ${ }^{15}$ Sharma et al described a case of right temporal otogenic brain abscess caused by Enterococcus faecalis in a 12-year-old male child. ${ }^{16}$ In contrast to these studies, our patient presented with expressive aphasia, which is a rare symptom of brain abscess. In addition, unlike the above cases, she had no primary source of infection.

Differential diagnoses for ring-enhancing lesions, as observed in our patient, include metastatic disease, brain abscess, cerebral infarction, lymphoma, high grade glioma, toxoplosmosis, demyelinating disease, radiation necrosis, or resolving cerebral contusion/hematoma. Brain imaging is indicated in any patient with rapid deterioration of mental status, in order to detect brain herniation or abscess rupture in the ventricular system, resulting in ventriculitis and often leading to hydrocephalus. ${ }^{17}$ Conversely, the lumbar puncture is contraindicated in patients with focal symptoms, signs of cranial nerve deficit, or papilloedema. Decompression of the cerebrospinal fluid (CSF) pressure below the tentorium, in association with asymmetric cerebral edema, results in brainstem herniation in $1.5 \%-30 \%$ of cases. ${ }^{18}$

Obtaining an accurate diagnosis for brain abscess can be challenging. A study by Gorgan et al found that half of patients were initially diagnosed inaccurately, and it took average of 7.2 days to receive a diagnosis of brain abscess. The reported morbidity for this cohort was $26.19 \%$, and mortality remained stable at $7.14 \% .{ }^{19}$ An MRI of the brain is more sensitive than CT scan for diagnosis of brain abscess. MRI is capable of differentiating ring-enhancing lesions due to brain abscess from neoplastic lesions, as abscess are usually hyperintense and neoplastic lesions are hypointense. ${ }^{20}$ Proton nuclear magnetic resonance spectroscopy can further differentiate abscess from cystic tumor. This technique allows for the detection of bacterial metabolic byproducts (e.g., lactate, acetate, and succinate) and neutrophil proteolysis (cytosolic amino acids). A study performed by Lai et al demonstrated the added value of spectroscopy and diffusion weighted imaging (DWI) to MRI in the diagnosis of intracranial cystic lesions. Specifically, spectroscopy and DWI raised the sensitivity and specificity of MRI for abscess from $61.9 \%$ and $60.9 \%$ to $95.2 \%$ and $100 \%$, respectively. ${ }^{21}$

A diagnosis of brain abscess is considered definite if bacterial organisms can be isolated from abscess pus or CSF cultures. However, Lu et al have previously reported that 19 out of 123 patients with brain abscesses were culturenegative. ${ }^{22}$ There were two cases reported by Yamamoto et al which had left frontal lobe abscess which were caused by S. intermedius. ${ }^{23}$

Factors influencing optimal management strategy for brain abscess include, size, location, number of abscesses, pathological phase, primary source of infection, probable causative pathogen, neurologic status, and performance status. Abscess recurrence or failure to improve is not uncommon after aspiration, and open craniotomy with excision is associated with a lower rate of recurrence and reaccumulation. ${ }^{24}$ If brain imaging does not show a central cavity in the abscess, careful consideration should be given to the choice between performing a stereotactic biopsy of the area of presumed cerebritis and administering empirical antimicrobial treatment with follow-up cranial imaging.

Retrospective analysis done by Helweg-Larsen et al the duration of antibiotics for brain abscess is prolonged, usually 4-8 weeks. ${ }^{25}$ Guidelines by the working party of the British Society in UK recommend 4-6 weeks if the abscess has been drained or excised and 6-8 weeks if the abscess is treated without drainage. ${ }^{26}$

Patients with multiple small $(<2 \mathrm{~cm})$ abscesses may respond to medical therapy alone. An abscess size $>2.5 \mathrm{~cm}$ in diameter has been recommended as an indication for neurosurgical intervention. ${ }^{1}$ Intravenousantibiotics should also be given for minimum of $6-8$ weeks, with a prolonged treatment recommended for immunocompromised patients. ${ }^{27,28}$ Ceftriaxone is the preferred agent, as it has excellent tissue penetration, and for these infections penicillin $\mathrm{G}$ has minimal inhibitory concentration of $<0.125 \mu \mathrm{g} / \mathrm{mL}$. If the patient is allergic or resistant to penicillin, vancomycin is the alternative drug of choice.

In our case, CT scan of the patient's head revealed a left temporal lobe mass-like lesion, with surrounding vasogenic 
edema, after which, the patient was prescribed a combined treatment of vancomycin, ampicillin, and ceftriaxone antibiotics. MRI of the brain further revealed a septated rim-enhancing mass lesion $5.5 \times 4.3 \mathrm{~cm}$ in the left temporal lobe, with bright restricted diffusion and diffuse surrounding vasogenic edema. Following stereotactic, left temporal craniotomy, with drainage and resection of abscess, the causative agent was determined to be $S$. intermedius. Treatment was then deescalated to intravenous ampicillin sulbactum, and the patient eventually made a full recovery.

Corticosteroids were not administered to this patient, and their use for brain abscess treatment is controversial. Steroids can retard the encapsulation process, increase necrosis, reduce antibiotic penetration into the abscess, increase the risk of ventricular rupture, and alter the appearance on CT scans because of contrast reduction. Steroid therapy can also produce a rebound effect when discontinued. Corticosteroids are recommended when a significant mass effect is visible on imaging, and the patient's mental status is depressed. Use of glucocorticoid therapy should be limited to patients with cerebral edema that are likely to herniate. ${ }^{29}$ Tremont-Lukats et al further showed that preventive treatment with antiseizure medications was not associated with a decrease in seizures. ${ }^{30}$

\section{Conclusion}

Brain abscess has a high mortality with numerous severe sequelae, and temporal lobe abscess associated with expressive aphasia is rare presentation. Here, we describe a unique case of temporal lobe abscess caused by $S$. intermedius in an immunocompetent adult presenting with expressive aphasia and no primary source of infection. Advanced imaging modalities may yield more accurate methods of differentiation of mass lesions in the brain. Biopsy of brain lesion with early initiation of appropriate antibiotics will change the outcome. A significant proportion of patients with appropriately treated abscess recover completely and can survive without significant neurologic damage.

\section{Acknowledgment}

The authors thank the patient for allowing them to use images for scientific purposes.

\section{Authors' contributions}

All authors contributed toward data analysis, drafting and revising the paper and agree to be accountable for all aspects of the work.

\section{Disclosure}

The authors report no conflicts of interest in this work.

\section{References}

1. Mamelak AN, Mampalam TJ, Obana WG, Rosenblum ML. Improved management of multiple brain abscesses: a combined surgical and medical approach. Neurosurgery. 1995;36(1):76-85.

2. Patel K, Clifford DB. Bacterial brain abscess. Neurohospitalist. 2014; 4(4):196-204.

3. Tseng JH, Tseng MY. Brain abscess in 142 patients: factors influencing outcome and mortality. Surg Neurol. 2006;65(6):557-562.

4. Brouwer MC, Coutinho JM, van de Beek D. Clinical characteristics and outcome of brain abscess: systematic review and meta-analysis. Neurology. 2014;82(9):806-813.

5. Tonon E, Scotton PG, Gallucci M, Vaglia A. Brain abscess: clinical aspects of 100 patients. Int J Infect Dis. 2006;10(2):103-109.

6. Antinori A, Larussa D, Cingolani A; Italian Registry Investigative NeuroAIDS. Prevalence, associated factors, and prognostic determinants of AIDS-related toxoplasmic encephalitis in the era of advanced highly active antiretroviral therapy. Clin Infect Dis. 2004;39(11):1681-1691.

7. Ruoff KL. Streptococcus anginosus ("Streptococcus milleri"): the unrecognized pathogen. Clin Microbiol Rev. 1988;1(1):102-108.

8. Jacobs JA, Pietersen HG, Stobberingh EE, Soeters PB. Bacteremia involving the "Streptococcus milleri" group: analysis of 19 cases. Clin Infect Dis. 1994;19(4):704-713.

9. Mishra AK, Fournier PE. The role of Streptococcus intermedius in brain abscess. Eur J Clin Microbiol Infect Dis. 2013;32(4):477-483.

10. GUTHOF O. [Pathogenic strains of Streptococcus viridans; streptocci found indental abscesses and infiltrates in the region of the oral cavity]. Zentralbl Bakteriol Orig. 1956;166(7-8):553-564. German.

11. Pecharki D, Petersen FC, Scheie AA. Role of hyaluronidase in Streptococcus intermedius biofilm. Microbiology. 2008;154(Pt 3):932-938.

12. Roche M, Humphreys H, Smyth E, et al. A twelve-year review of central nervous system bacterial abscesses; presentation and etiology. Clin Microbiol Infect. 2003;9(8):803-809.

13. Stewart TA, Carter CS, Seiberling K. Temporal lobe abscess in a patient with isolated sphenoiditis. Allergy Rhinol (Providence). 2011;2(1):40-42.

14. Chen PT, Ho KY, Tai CF, Su KJ, Lin IF, Kuo WR. Otogenic brain abscess - a case report. Kaohsiung J Med Sci. 2000;16(3):162-165.

15. Guzman C, Isaac Y, Quinton G. Management and treatment of otogenic temporal lobe abscess secondary to mastoiditis: a case report and review of literature. J Case Rep Med. 2013.

16. Sharma S, Malhotra S, Bhatia NJK, Wilson A, Hans C. Right temporal otogenic brain abscess by Enterococcus faecalis - a rare case report. Int J Curr Microbiol App Sci. 2014;3(3):101-104.

17. Brouwer MC, Tunkel AR, McKhann GM 2nd, van de Beek D. Brain abscess. N Engl J Med. 2014;371(5):447-456.

18. Tattevin P, Bruneel F, Clair B, et al. Bacterial brain abscesses: a retrospective study of 94 patients admitted to an intensive care unit (1980 to 1999). Am J Med. 2003;115(2):143-146.

19. Gorgan M, Neacsu A, Bucur N, et al. Brain abscesses: management and outcome analysis in a series of 84 patients during 12 year period. Romanian Neurosurg. 2012;19(3):175-182.

20. Friedlander RM, Gonzalez RG, Afridi NA, Pfannl R. Case records of the Massachusetts General Hospital. Weekly clinicopathological exercises. Case 16-2003. A 58-year-old woman with left-sided weakness and a right frontal brain mass. N Engl J Med. 2003;348(21):2125-2132.

21. Lai PH, Hsu SS, Ding SW, et al. Proton magnetic resonance spectroscopy and diffusion-weighted imaging in intracranial cystic mass lesions. Surg Neurol. 2007;68(Suppl 1):S25-S36.

22. Lu CH, Chang WN, Lin YC, et al. Bacterial brain abscess: microbiological features, epidemiological trends and therapeutic outcomes. QJM. 2002;95(8):501-509.

23. Yamamoto M, Fukushima T, Ohshiro S, et al. Brain abscess caused by Streptococcus intermedius: two case reports. Surg Neurol. 1999;51(2): 219-222.

24. Gadgil N, Patel AJ, Gopinath SP. Open craniotomy for brain abscess: a forgotten experience? Surg Neurol Int. 2013;4:34.

25. Helweg-Larsen J, Astradsson A, Richhall H, Erdal J, Laursen A, Brennum J. Pyogenic brain abscess, a 15 year survey. BMC Infect Dis. 2012;12:332. 
26. Infection in Neurosurgery Working Party of the British Society for Antimicrobial Chemotherapy. The rational use of antibiotics in the treatment of brain abscess. Br J Neurosurg. 2000;14(6): 525-530.

27. Jansson AK, Enblad P, Sjölin J. Efficacy and safety of cefotaxime in combination with metronidazole for empirical treatment of brain abscess in clinical practice: a retrospective study of 66 consecutive cases. Eur J Clin Microbiol Infect Dis. 2004;23(1):7-14.
28. Takeshita M, Kagawa M, Izawa M, Takakura K. Current treatment strategies and factors influencing outcome in patients with bacterial brain abscess. Acta Neurochir (Wien). 1998;140(12):1263-1270.

29. Quartey GR, Johnston JA, Rozdilsky B. Decadron in the treatment of cerebral abscess. An experimental study. J Neurosurg. 1976;45(3):301-310.

30. Tremont-Lukats IW, Ratilal BO, Armstrong T, Gilbert MR. Antiepileptic drugs for preventing seizures in people with brain tumors. Cochrane Database Syst Rev. 2008;(2):CD004424.

\section{Publish your work in this journal}

The International Medical Case Reports Journal is an international, peer-reviewed open-access journal publishing original case reports from all medical specialties. Previously unpublished medical posters are also accepted relating to any area of clinical or preclinical science. Submissions should not normally exceed 2,000 words or
4 published pages including figures, diagrams and references. The manuscript management system is completely online and includes a very quick and fair peer-review system, which is all easy to use. Visit http://www.dovepress.com/testimonials.php to read real quotes from published authors.

Submit your manuscript here: https://www.dovepress.com/international-medical-case-reports-journal-journal 